\title{
VLSI Implementation of Low Power Area Efficient Fast Carry Select Adder
}

\author{
J. Eric Clapten \\ Department of Electronics and \\ Communication Engineering, \\ Dhanalakshmi Srinivasan \\ College of Engineering, \\ Coimbatore, India.
}

\author{
E. Konguvel \\ Department of Electronics and \\ Communication Engineering, \\ Dhanalakshmi Srinivasan \\ College of Engineering, \\ Coimbatore, India.
}

\author{
M. Thangamani \\ Department of Electronics and \\ Communication Engineering, \\ Dhanalakshmi Srinivasan \\ College of Engineering, \\ Coimbatore, India.
}

\begin{abstract}
Carry Select Adder (CSLA) is one of the speedest adder utilized as a part of numerous computational frameworks to perform quick number-crunching operations. The Carry select adder utilizes an effective plan by imparting the Common Boolean logic (CLB) term. The modified CSLA architecture building design has created utilizing Binary to Excess-1 converter (BEC). This paper introduces an unique method that replaces the BEC using common Boolean logic. Experimental analysis illustrates that the proposed architecture achieves advantages in terms of speed, area consumption and power.
\end{abstract}

\section{General terms}

Common Boolean Logic, VLSI

\section{Keywords}

Carry Select Adder, Area-Efficient, BEC

\section{INTRODUCTION}

Outline of area and power effective rapid information rationale frameworks are a standout amongst the most considerable areas of exploration in VLSI framework plan. In gadgets applications adders are most generally utilized. In computerized adders, the speed of expansion is restricted when needed to engender a carry through the snake. The aggregate for every bit position in a elementary adder is produced consecutively strictly when the past bit position are summed and carry spread into the following position [1].

The CSLA is utilized as a part of numerous computational frameworks to allay the issue of carry spread postpone by free era different conveys and afterward select a carry to create the aggregate. On the other hand, the CSLA is not an area productive on the grounds that it utilizes different sets of Ripple Carry Adders(RCA) to produce halfway total and carry by considering carry include as $\mathrm{C}_{\mathrm{in}}=0$ and $\mathrm{C}_{\mathrm{in}}=1$, then the last summation and carry are chosen by the multiplexers. The Carry select adders are delegated Linear Carry select adders and Square-root carry select adders [2]. The fundamental square-root Carry Select adders has a double ripple carry adder with 2:1 multiplexer, the principle detriment of consistent CSLA is the expansive area because of the different sets of ripple carry adder.

In this paper, modified CSLA using BEC has introduced to reduce area and power consumption with slight increase in delay. The basic idea of the proposed architecture is that which replaces the BEC by common Boolean logic with enable signal[9]. The proposed architecture reduces the area, delay and power[10]. This paper is organized as follows; section 2 review the related works and section 3 presents the detailed structure of proposed methodology. Results are analyzed in the section 4 . Section 5 concludes the paper[11].

\section{LITERATURE SURVEY}

The author introduced a carry select adder (CSL) to reduce the delay as compared with the other existing adders. This adder has a deepness of knowledge in logarithmic gate to design any of the formation an adder's family. The ripple-carry adder (RCA) is used in CSL which leads to less performance. So one of the fastest adder called Carry Select Adder (CSLA) is used to carry out the arithmetic operation as earlier. The CSLA is a best adder that provides optimal result in VHDL execution and it may effectively diminish the CSLA parameters. The simulation result shows that the modification 32-b CSLA (CSLA) structural design gives better result as compared with the other techniques is studied in [3].

In [4] a hybrid full adder with low power multipliers is used. In [5] the Ripple carry adder shows the evidence of unsophisticated designs of the circuit but it speed is slow. The carry look ahead adder (CLA) is one of the best one but it occupies large area. CSA is act as a concession between two adders like Ripple carry adder and carry look ahead. 32-Bit Multiplier with a CLA and a 32-bit Multiplier with a RCA are implemented in VHDL to analyze the performance is studied in [6]. In [7] the author presents a square-root CSLA (SQRT CSLA) which reduces the area and the power as it is compared with the other techniques to evaluate the performance.In [8] proposed a BEC method to minimize the carry propagation delay in final stage of carry save adder.

\section{PROPOSED METHODOLOGY}

The system analysis deals with Binary to Excess-1 Conversion and the Common Boolean Logic method.

In proposed methodolocy, a common Boolean logic term is shared by an area-efficient carry select adder to eliminate the duplicated adder cells in the conventional carry select adder. In this way it achieves low Power and saves many transistor counts. By analyzing the a single-bit fulladder truth table the output of summation signal is calculated by the carry-in signal is logic " 0 " or as the inverse signal of itself as the carry-in signal is logic "I ". As illustrated as two dotted circles in the truth table of Table 1 [3]. By sharing the common Boolean logic term in summation generation, a proposed carry select adder design is illustrated in Fig 1[12]. To share the common Boolean logic term, it only needs to implement one OR gate with one INV gate to generate the Carry signal and summation signal pair. Once the carry-in signal is ready, then select the correct carry-out output according to the carry-in signal logic state[13]. As compared with the Modified Carry Select adder, the proposed CSLA is little bit faster, but the speed is nearly equal to the Regular CSLA. The delay time in our proposed adder design is also proportional to the bit number $\mathrm{N}$; however, the delay time of multiplexer is shorter 
than that of full adder. The Proposed CSLA is Area efficient $\&$ low power, but the speed equal to the Regular CSLA.

Table 1. The truth table of single bit FA and single-bit fulladder with common Boolean logic

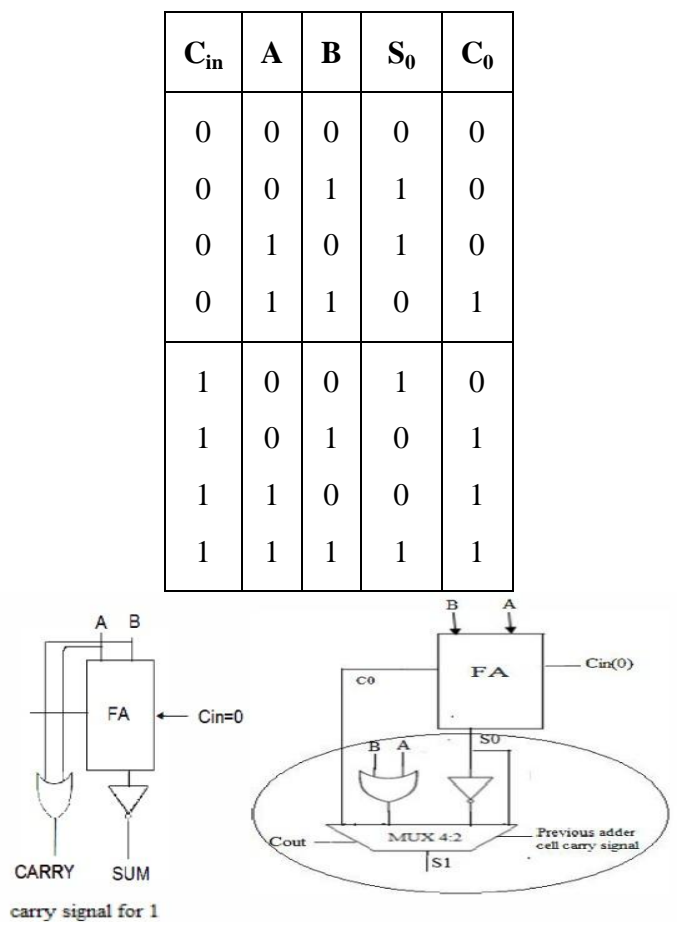

Fig 1: Single bit FA with Common Boolean Logic

\subsection{Modified CSLA Architecture}

This method replaces the BEC add one circuit by Common Boolean Logic. The output of full adder waveform for carry in signal is ' $I$ ' and it generates the carry signal and summation by just using an OR gate and INV[18]. It is shown in fig 2 .

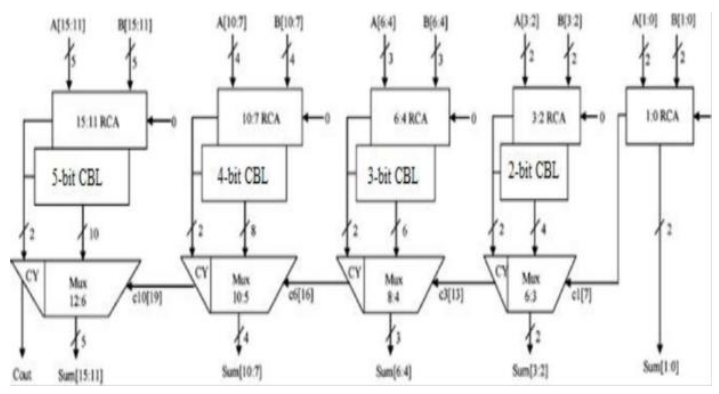

Fig 2: 16-bit SQRT CSLA using CBL

The carry signal and summationfor FA which has Cin=l, it is generated by the INV and OR gate. With the help of multiplexer, we can predict the correct output result according to the logic state of each carry-in signal. Internal structure of the group 3 of Proposed CSLA is shown Fig 3. A Manual counting of number of gates is used for group 3 is equal to 36 (full adder, half adder, and multiplexer, not, or gate)[12]. One input to the mux goes from the RCA block with $\mathrm{Cin}=\mathrm{O}$ and other input from the CBL.

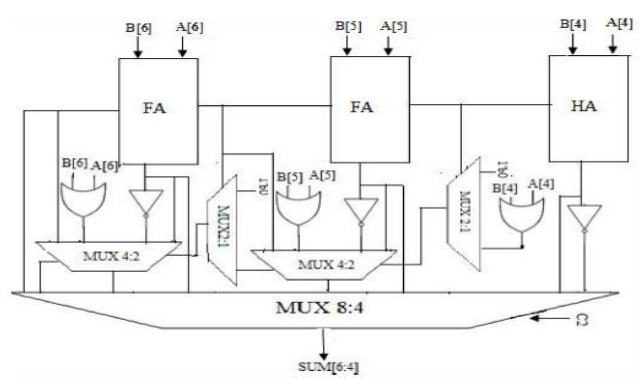

Fig 3: Group 3 using CBL

The Group 3 performed a three bit addition which are A [4] with $\mathrm{B}[4], \mathrm{A}[\mathrm{S}]$ with $\mathrm{B}[\mathrm{S}]$ and $\mathrm{A}[6]$ with $\mathrm{B}[6]$. This is done by I half adder (RA) and two full adder (FA). The CBL block has a 4:2 multiplexer to select the appropriate carryout and summation signal for Carry-in signal ' I '. Through 2: I multiplexer the carry signal is propagate to the next adder cell. The 6:3 multiplexer and 4:2 multiplexer is the combination of 2: I multiplexer[14].

\section{PERFORMANCE EVALUATION}

The proposed design used in this research work has been developed using synthesized in Cadence RTL compiler using typical libraries of TSMC 0.18 um technology Verilog- HDL. The synthesized Verilog netlist and their respective design constraints file (SDC) are imported to Cadence SoC Encounter and are used to generate automated layout from standard cells and placement and routing. Parasitic extraction is performed using Encounter's Native RC extraction tool and the extracted parasitic RC (SPEF format) is back annotated to Common Timing Engine in Encounter platform for static timing analysis. The performance of the proposed approach is evaluated using the performance metrics such as Delay, area and power. Several real time implementation strategies were discussed in [15],[16] and [17].

\subsection{Power Analysis}

The power analysis has been done in modelsim by the method of Common Boolean Logic and the output waveform is shown in fig 4 .

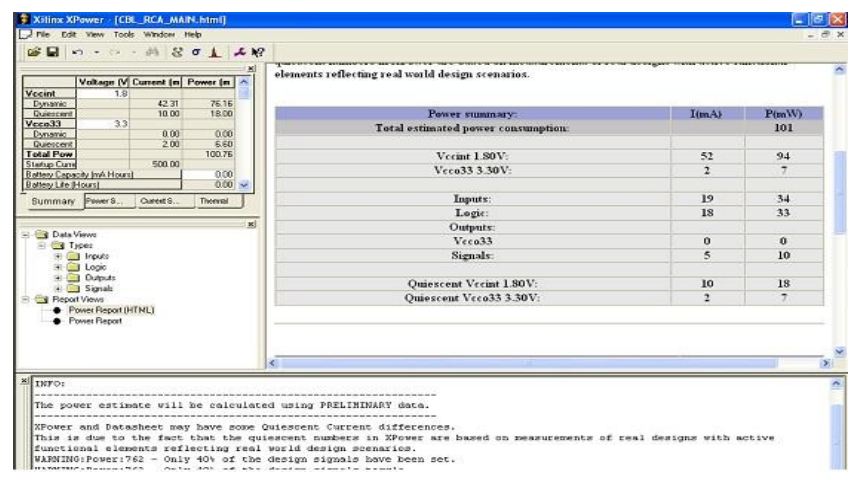

Fig 4: Analysis of Power by CBL Method

\subsection{Delay Analysis}

The delay analysis has been done in modelsim by the method of Common Boolean Logic and the output obtained is shown in fig 5 . 


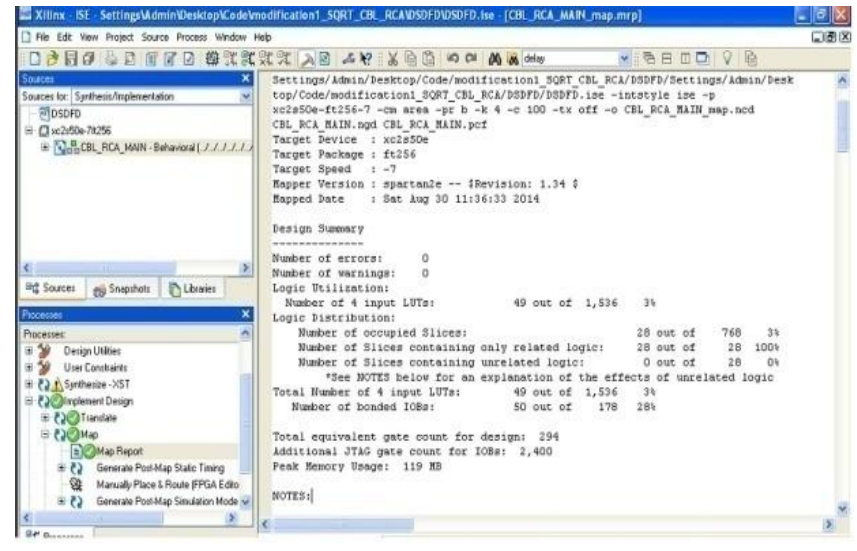

Fig 5: Analysis of Delay by CBL Method

\subsection{Area Analysis}

The area analysis has been done in modelsim by the method of Common Boolean Logic and the output obtained is shown in fig 6.

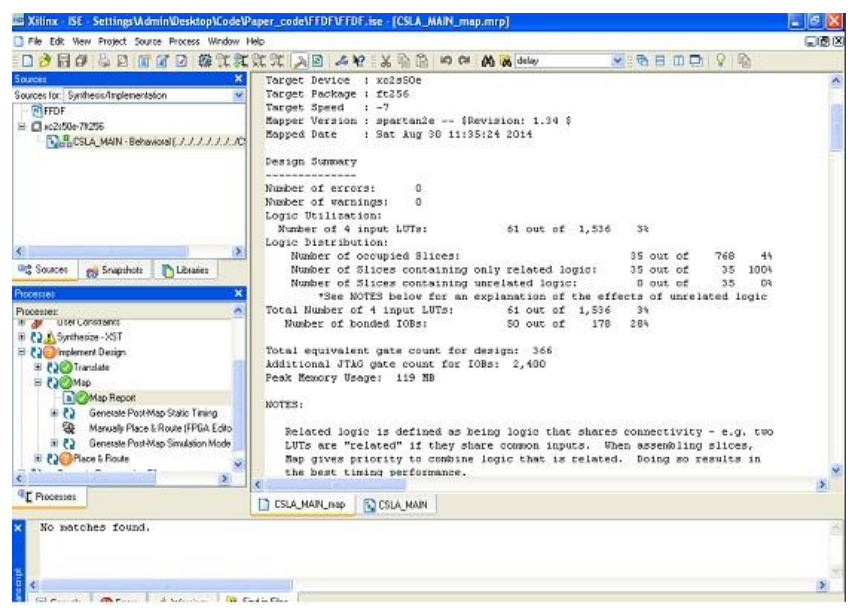

Fig 6: Analysis of Area by CBL Method

\subsection{Comparison}

The area, delay and power analysis of carry select adder with $\mathrm{CBL}$ approach has been performed and the results are shown in the table 2.

Table 2. Comparison table of BEC-1 and CBL method

\begin{tabular}{|c|c|c|}
\hline PARAMETERS & EXISTING & PROPOSED \\
\hline $\begin{array}{c}\text { NO OF GATES } \\
\text { USED }\end{array}$ & 366 & 294 \\
\hline DELAY (ns) & 24.822 & 18.268 \\
\hline POWER(mW) & 108 & 101 \\
\hline
\end{tabular}

\section{CONCLUSION}

In this paper analysed the logic operations involved in the conventional and BEC-based CSLAs to study the data dependence and to identify redundant logic operations. And also eliminated all the redundant logic operations of the conventional CSLA and proposed a new logic formulation for the CSLA. The modified SQRT CSLA reduces the area and power when compared to regular CSLA with increase in delay by the use of Binary to Excess-I converter. This paper proposes a scheme which reduces the delay, area consumption and power than regular and modified CSLA by the use of Common Boolean Logic. In future work, It would be interesting to test the design of the proposed 128-b SQRT CSLA by Quantum dot Cellular Automata(QCA) method. Inbrief, we propose another adder, that achieves all conditions of competitors and accomplishes the best area-delay tradeoff by using a majority gate. The above preferences are obtained by utilizing an overall area/space to the less expensive plans as known in the literature.

\section{REFERENCES}

[1] B.Ramkumar and Harish M Kittur, "Low power and area efficient carry select adder", IEEE Transactions on Very Large Scale Integration (VLSI) Systems, vol. 20, no. 2, pp. 371-375, Feb 2012.

[2] J. M. Rabaey, Digital Integrated Circuits-A Design Perspective, Upper Saddle River, NJ: Prentice-Hall, 2001.

[3] Shalemraju Kolasani, K. Hanumantha Rao \& T. Malyadri," A Novel Designing Approach for high speed carry Select adder", International Journal of Computer Science and Electrical Engineering (IJCSEE) ISSN No. 2315-4209, Vol-1, Iss-2, 2012.

[4] Z.Abid, H. El-Razouk and D.A. El-Dib, "Low power multipliers based on new hybrid full adders", Microelectronics Journal, Volume 39, Issue 12, Pages 1509-1515, 2005.

[5] A. Tyagi, "A reduced area scheme for carryselect adders", IEEE Trans. on Computer, vol. 42, pp. 11631170, 1993.

[6] Hasan Krad and Aws Yousif Al-Taie, "Performance Analysis of a 32-Bit Multiplier with a Carry-Look-Ahead Adder and a 32-bit Multiplier with a Ripple Adder using VHDL", Journal of Computer Science 4 (4): 305-305, 2005.

[7] B.Ramkumar and Harish M Kittur, "LowPower and Area-Efficient Carry Select Adder", IEEE Transactions on Very Large Scale Integration (VLSI) systems, vol. 20, no. 2, February 2012.

[8] B.Ramkumar, H.M. Kittur, and P. M. Kannan, -ASIC implementation of modified faster carry save adder,\| Eur. J. Sci. Res., vol. 42, no. 1, pp. 53-55, 2010.

[9] http://www.ijmra.us/project\%20doc/IJMIE_MAY2012/IJ MRA-MIE1 152.pdf

[10] http://www.ijesit.com/Volume\%202/Issue\%204/IJESIT2 01304_49.pdf

[11] http://airccse.org/journal/ijdms/papers/3411ijdms01.pdf

[12] http://www.slideshare.net/ijeraeditor/eq36876880

[13] http://www.academia.edu/10177958/An_Area_Efficient_ Carry_Select_Adder_for_Signal_Processing_Application $\mathrm{s}$

[14] http://www.jatit.org/volumes/Vol64No3/18Vol64No3.pd $\mathrm{f}$

[15] J.Raja, M.Kannan, VLSI implementation of high throughput MIMO OFDM transceiver for $4^{\text {th }}$ Generation systems. ,international journal of Engineering and materials sciences,Vol. 19 ,October 2012, pp. 307-312. 
International Journal of Computer Applications (0975 - 8887)

Volume 115 - No. 6, April 2015

[16] E. Konguvel, J. Raja, M. Kannan, A Low Power VLSI Implementation of 2X2 MIMO OFDM Transceiver with ICI-SC Scheme, International Journal of Computer Applications (0975 - 8887) Volume 77 - No.5, September 2013.

[17] A.Amjadha, E. Konguvel, J. Raja, Design of Multipath Delay Commutator Architecture based FFT Processor for $4^{\text {th }}$ Generation System International Journal of Computer Applications (0975 - 8887) Volume 89 - No 12, March 2014.

[18] Basant Kumar Mohanty and Sujit Kumar Patel, AreaDelay-Power Efficient Carry Select Adder" IEEE Transactions on Circuits and Systems-II: Express briefs, Vol. 61, no. 6,June 2014. 